\title{
Pioneer craniotomy at Babcock University Teaching Hospital: Anaesthetic management
}

\author{
F.A.O. Oguntade', D.A. Aderinto' ${ }^{2}$ O.F. Salami' \\ 'Department of Anaesthesia and Intensive Care, Babcock University Teaching Hospital Ilisan-Remo, Ogun State, Nigeria \\ ${ }^{2}$ Department of Anaesthesia, University College Hospital, Ibadan
}

\begin{abstract}
Glioblastoma multiforme is the highest grade glial tumour and most common in the astrocytic line. This usually requires craniotomy for tumour biopsy, relief of intracranial pressure and reduction in tumour mass as much as possible. In the anaesthetic management of patient for craniotomy, emphasis should be on the provision of safe and optimal preoperative conditions, preservation of neurocognitive functions and a rapid high quality recovery. Maintenance of adequate cerebral
\end{abstract}

perfusion pressure (CPP), normal intra-cranial pressure (ICP) and cerebral oxygenation $\left(\mathrm{CMRO}_{2}\right)$ is the mainstay of neuro-anaesthesia and requires a thorough understanding of the physiology of the central nervous system (CNS). We report a case of a 59 year old man with glioblastoma multiforme and features of raised intracranial pressure that successfully underwent craniotomy and decompression of the tumour.

Corresponding author: Dr D.A. Aderinto, Department of Anaesthesia, , University College Hospital, Ibadan. Email: davederinto@yahoo.com

\section{INTRODUCTION}

The diagnosis of glioblastoma multiforme is usually in the $5^{\text {th }}-6^{\text {th }}$ decade of life by non-invasive imaging (CT scan and or MRI) in a patient who presented with headache, seizures, mental status changes or focal neurological deficits ${ }^{1}$. Better understandings of the effects of anaesthesia on the central nervous system (CNS) and improvement in anaesthetic techniques have contributed immensely to the improved outcomes seen in modern neurosurgery.

The brain is very vulnerable to ischaemic injury because of its relatively high oxygen consumption and near-total dependence on aerobic glucose metabolism. Interruption of cerebral perfusion, metabolic substrate or severe hypoxaemia rapidly results in functional impairment ${ }^{2}$. Dexamethasone is prescribed routinely to reduce cerebral oedema in patients with brain tumour.

This significantly increases blood glucose concentration in non- diabetic patient, resulting in metabolic and cerebral ischaemic effect. Neurosurgical monitoring aims to detect changes in the cerebral haemodynamic, oxygenation and neuronal function. Intraoperative electrophysiological monitoring may help to prevent postoperative deficit.

A speedy recovery is desired to allow prompt neurological assessment and early detection of complications that require immediate intervention. Neuro-anaesthesia can be quite challenging to the anaesthetist, especially when the procedure is being performed in a centre that has no pre-existing neurosurgical facilities and manpower like ours. We present a 59 year old man with glioblastoma multiforme and features of raised intracranial pressure (ICP) who underwent an elective craniotomy and decompression of tumour in our institution as a maiden neurosurgical case.

\section{CASE REPORT}

A 59 year old man, who presented with two month history of recurrent headache and ear fullness, associated with blurring of vision, tremors, and loss of consciousness/syncope, right sided body weakness. No history of vomiting or any other comorbidities, but has mild weight loss.

General examination was essentially normal. Systemic examination was also normal, except for mild prostate enlargement on rectal examination and significant papilloeodema at fundoscopy. Magnetic resonance imaging showed an intra-cranial lesion of a large ill-defined heterogeneous enhancing soft tissue mass with area of necrosis and haemorrhage involving the splenium of the corpus callosum and left deep periventricular parieto-occipital lobe white mater with moderate oedema and significant midline shift to the right. A diagnosis of intra-cranial tumour (? Glioblastoma) was made. Patient was scheduled for craniotomy plus tumour excision. He was also commenced on dexamethasone $4 \mathrm{mg} 6$ hourly and phenytoin $1.5 \mathrm{mg}$ daily.

Airway assessment was mallampati II, PCV of $36 \%$, Liver function test and Electrolyte and urea were within normal limits. A chest and abdominalpelvic CT scan yielded no additional information. Lung function test done was normal. An assessment of ASA IV was made.

Being the maiden neurosurgical / 
neuroanaesthesia case, a conference was held with all stake holders physically present a week before surgery. These involved the Surgeons, Anaesthetists, Perioperative and ICU nurses, and the representative of the biomedical department.

Two weeks after presentation patient was wheeled into the theatre. In the supine position baseline parameters showed tachycardia of 122 beats/min, elevated blood pressure (BP) 164/98 $\mathrm{mmHg}$, oxygen saturation $\left(\mathrm{SPO}_{2}\right) 97 \%$ and respiratory rate (RR) 18cycles/ min. Intravenous access in both arms was established with size 18G cannula and $0.9 \%$ Normal Saline infused at $100 \mathrm{mls} /$ hour. Patient was premedicated with intravenous fentanyl $100 \mu \mathrm{g}$, ondansetron $8 \mathrm{mg}$. Preoxygenation was carried out for 5 minutes. Intubation with size $7.00 \mathrm{~mm}$ re-enforced cuffed endotracheal tube was facilitated with I.V pancuronium $8 \mathrm{mg}$ after induction with total dose of $500 \mathrm{mg}$ sodium thiopentone.

Correct tube placement was confirmed and patient connected to the anaesthetic machine and manually ventilated. Immediate post intubation parameters were, PR 118mmHg, BP 130/100 $\mathrm{mmHg}$, end-tidal carbon dioxide $\left(\mathrm{ETCO}_{2}\right) 35 \mathrm{mmHg}$. The eyes were padded and tapped. Patient was positioned in the semil management lateral position (Janetta position), with head elevated to about 15-30 degree. Mechanical ventilation was instituted with the following parameters: IPPV without positive end-expiratory pressure (PEEP), FiO2 100\% (6L/min), tidal volume $500 \mathrm{mls}$, RR of 10cycles/minute, I:E of $1: 2$ and maintained on isoflurane $1-2 \%$ in $100 \%$ oxygen.

Patients scalp was infiltrated with about 20 mls of 1:200,000 epinephrine. I.V morphine 10 mg was given intra-operatively and $300 \mathrm{mls}$ of $20 \%$ mannitol was infused during craniotomy. 1\% Propofol was infused at the rate of $10 \mathrm{mg} / \mathrm{kg} / \mathrm{hr}$ initially, and then gradually reduced to $5 \mathrm{mg} / \mathrm{kg} / \mathrm{hr}$ based on the patient's blood pressure. Isoflurane was reduced to $0.5 \%$ dial over time in response to the effect of the propofol infused. Surgery lasted 4 hrs 15 minutes, during which patient also had I.V pancuronium 'by the clock' to maintain muscle relaxation. A total of 4.5 litres of $0.9 \%$ normal saline and $500 \mathrm{mls}$ of whole blood was infused. A total of 1,770 mls of urine was drained, with an estimated blood loss of 1.8 litres.

Heamodynamic stability was maintained throughout the procedure, a mean arterial pressure of greater than $80 \mathrm{mmHg}$ was targeted. A single incidence of hypotension which occurred 150 minutes intra-operatively (BP 75/40 $\mathrm{mmHg}$ ), estimated blood loss at the time was about 1.2 litres. Blood pressure normalized following transfusion of one unit of blood when rapid saline infusion and temporary discontinuation of propofol infusion failed. ETCO2 was maintained at 31 to $35 \mathrm{mmHg}$ throughout surgery.

At the last stitch on the scalp, propofol was turned off. Isoflurane was turned off after adequate oral suctioning post dressing. Ventilation was changed to manual mode with patient still on $100 \%$ oxygen. Residual neuromuscular block was reversed when curare cleft was noticed on capnography tracings. He was extubated deep after adequate muscle activity was confirmed clinically. Patient was transferred to the ICU post extubation. Supplemental oxygen was administered via the face mask and continuous ECG and SPO2 monitored during transportation. On arrival at the ICU, GCS was 15 , and patient moved all the 4 limbs in response to command. There was no pain at all and vital signs were within normal limits.

\section{DISCUSSION}

Glioblastoma multiforme is a tumour of the brain tissue arising from astrocytes which are the supporting tissue of the brain. Glioblastomas represent $15.4 \%$ of all primary brain tumours and $60-75 \%$ of all astrocytomas. Incidence increases with age with more male preponderance. $9 \%$ of all childhood tumours are glioblastomas ${ }^{1}$. The tumour is highly malignant, reproduces quickly and is supported by a large network of blood vessels. It is generally found in the cerebral hemispheres of the brain, but can be found in any part of the brain and spinal cord. In this index case the tumour was found in the corpus callosum and the periventricular white mater area of the brain. The tumour could either be primary, in which case it is faster in clinical presentation and it is the most common. The secondary type is more insidious in onset, though also aggressive. It is commoner in less than 45years of age and made up of only $10 \%$ of the glioblastomas ${ }^{1}$. In our patient no secondary lesion was found.

Due to its rapid increase in size, the common symptoms are those due to increased intracranial pressure (ICP). These symptoms include headache, nausea, vomiting and drowsiness. They may also present with other symptoms like hemiparesis, memory impairment, speech difficulty and visual changes, depending on the location of the tumour.

The first step in the management of the tumour is a procedure to make a diagnosis via tissue biopsy and simultaneously reliving intracranial pressure and safely debulking the tumour as much as possible through surgery. In our patient, the signs of raised ICP were present as earlier documented. Hence craniotomy was carried out to achieve the above stated purposes ${ }^{1}$. 
The anaesthetic consideration and goal were essentially to limit further increase in the ICP, put measures in place to reduce the ICP, reducing cerebral metabolism, use anticonvulsant agents while avoiding epileptogenic agent.

Although both sodium thiopentone and propofol have potential to reduce cerebral blood flow (CBF), cerebral metabolic rate (CMR) and reduce ICP. The choice of sodium thiopentone over propofol was because of its proven ability to reduce oxygen demand and its potent anticonvulsant properties without causing much hypotension associated with propofol at induction of anaesthesia ${ }^{3}$. Fentanyl was administered based on its effect on reducing the cardiovascular response to laryngoscopy and its deleterious effect on ICP as documented by several authors.

Preventing further increase in ICP was achieved using different techniques; Firstly, preventing increase in venous pressure was achieved by preventing obstruction of the neck veins by securing the endotracheal tube (ETT) with a tape and not with tube ties, lateral tilt of the shoulder with bolster's support in the same direction of neck rotation during patent positioning (semilateral position) and 15-30 degree head up tilt to enhance venous return. Coughing and straining on the ETT was avoided by adequate neuromuscular paralysis during the period of surgery. The patient was also extubated deep at the end of anaesthesia, bearing in mind the possible complications that may occur e.g. hypoventilation and upper airway obstruction. Ondasetron was also administered to prevent PONV that may lead to straining postoperatively.

Secondly, preventing further cerebral edema by using isotonic solution and not hypotonic solution, in this case normal saline was used throughout the surgery as maintenance fluid. This is because fluid flux across the blood-brain barrier is dependent on plasma osmolality and not the oncotic pressure. The reduction in serum osmolarity can results in oedema of normal and abnormal brain tissue ${ }^{4}$. Therefore fluids that are metabolized to free water were avoided e.g. glucose containing fluids. Though normal saline has the advantage of slightly been hyperosmolar compared to the plasma, it may cause hyperchloremic metabolic acidosis when administered in large volumes ${ }^{5}$. Ringer's lactate is slightly hypo-osmolar fluid with potential of decreasing the plasma osmolarity leading to cerebral oedema ${ }^{4}$.

Lastly, prevention of increase in CBF was achieved by avoiding hypoxia, hypercarbia, hypertension and hyperthermia intraoperatively. Good oxygenation, adequate analgesia and anaesthesia depth was ensured throughout the surgical period. Anaesthetic agents that are associated with raised ICP were avoided e.g. suxamethonium, ketamine and nitrous oxide. Where outright avoidance is not possible the best agent in the group was used e.g. isoflurane as an inhalational agent instead of halothane which causes markedly raised CBF and raised ICP. To further reduce the concentration of inhalational agent used intraoperatively and its consequent effect, continuous intravenous anaesthetic (propofol) was used. Propofol is noted for its neuroprotective ability.

Effort was also focused on reducing the ICP in this patient using different interventions. These interventions included: (1) Reduction in cerebral edema by administration of $20 \%$ mannitol at $1 \mathrm{~g} / \mathrm{kg}$ about 15 minutes before the dura matter was opened. (2) Moderate hyperventilation aimed at ETCO $_{2}$ of about $30-35 \mathrm{mmHg}$. An arterial carbondioxide tension (PaCO2) measurement would have been more appropriate but for non availability of an indwelling arterial catheter for sampling. The head up tilt will also help in reducing the ICP due to reduction in the central venous pressure associated with head up position.

Our monitoring was majorly limited to the non-invasive monitored parameters (NIBP, ECG, Pulse oximetry, Temperature and the end tidal C02). The patient was catheterized to monitor urinary output, prevent urinary retention and prevent contamination of the theatre environment. The non-availability of invasive monitors for the pressures i.e. arterial, central venous pressure and the cerebral perfusion pressure was a limitation during the care of this patient. Regular communication between the neurosurgical team and the anaesthetists, and prompt measurement/interpretation of the non-invasive parameters we employed followed by appropriate intervention provided an optimal peri-operative care.

The aim of care during the termination of anaesthesia is to prevent arterial hypertension that may lead to intracranial bleeding, haematoma formation and oedema ${ }^{6,7}$. This was achieved by appropriate timing of the discontinuation of the various anaesthetic used. Hence our patient had a smooth emergence and extubation. Other options available in preventing coughing and straining on the tube include the use of vasoactive agents like labetelol ${ }^{8}$, and other drugs like dexmedetomidine ${ }^{9}$ and intravenous lidocaine administration.

\section{CONCLUSION}

We were able to successfully manage the index patient by ensuring safe and optimal perioperative conditions that preserved neuro- 
cognitive functions and offered a rapid high quality recovery. Anaesthetists in developing countries with limited resources can provide optimal patient care by following basic principles of neuro-anaesthesia.

\section{REFERENCES}

1. American Brain Tumor Association: Glioblastoma and Malignant $\begin{array}{lllllllllllllllllll}A & s & t & r & o & c & y & t & o & m & a\end{array}$. http://www.abta.org/secure/glioblast oma-brochure.pdf

2. Morgan AG, Mikhail MS, Murray MJ: Clinical Anesthesiology, fourth edition; Lange Medical Books/McGraw-Hill, USA, 2006.

3. Wodey E, Chonow L, Beneux X, et al: Haemodynamic effects of propofol vs $\mathrm{th}$ i o pental i n i n f n t s: a n echocardiographic study. British Journal of Anaesthesia 82 (4): 516-20 1999.

4. Tommasino C, Moore S, Todd MM: Cerebral effects of isovolemic hemodilution with crystalloid or colloid solutions. Crit Care Med 16:862-868,
1988.

5. Kellum JA: Saline-ind u c ed hyperchloremic metabolic acidosis. Crit Care Med 30:259-261, 2002.

6. Kalfas IH, Little JR: Postoperative hemorrhage: A survey of 4992 intracranial procedures. Neurosurgery 23:343-347, 1988.

7. Johansson B, Li CL, Olsson Y, et al: The effect of acute arterial hypertension on the blood-brain barrier to protein tracers. Acta Neuropathol (Berl) 16:117-124, 1970.

8. Grilo P, Bruder N, Auquier $\mathrm{P}$ et al: Esmolol blunts the cerebral blood flow velocity increase during emergence from anesthesia in neurosurgical patients. Anesth Analg 96: 1145-1149, 2003.

Tanskanen PE, Kytta JV, Randell TT, et al: Dexmedetomidine as an anaesthetic adjuvant in patients undergoing intracranial tumour surgery: A double blind, randomized and placebo-controlled study 